JOURNAL OF ENGINEERING SCIENCES

ЖУРНАЛ ІНЖЕНЕРНИХ НАУК

ЖУРНАЛ ИНЖЕНЕРНЫХ НАУК

Web site: http://jes.sumdu.edu.ua

\title{
Static calculation of the dynamic deflection elements for separation devices
}

\author{
Pavlenko I. V. , Liaposhchenko O. O., Demianenko M. M., Starynskyi O. Ye.
}

Sumy State University, 2 Rymskogo-Korsakova St., 40007, Sumy, Ukraine

\section{Article info:}

Paper received:

The final version of the paper received:

Paper accepted online:
September 14, 2017

December 2, 2017

December 4, 2017
*Corresponding Author's Address:

i.pavlenko@omdm.sumdu.edu.ua

\begin{abstract}
The following paper considers the influence of acoustic oscillations on multiphase flows on their suspended particles, which can be destroyed or coagulated by vibrations. Considering this, the method of extension of application range of the dynamic separation element as vibrocoagulants due to the use of hydroaeroelasticity phenomena, namely flutter, has been proposed. There were considered the problems of development an engineering method for calculating dynamic separation elements, the main of which is the analytical solution of the hydroaeroelasticity problem. This work takes the first step to its development, considering the previous elastic elements deformation that has a significant effect on the flutter frequency. The state of their static equilibrium was conducted with the use of analytical dependencies of the finite element method. The bimodal finite elements with six degrees of freedom were used for dynamic deflection elements. As the result, there was determined the stiffness of pre-deformed plates and their maximum and minimum possible deflections. The functions of the median surface deflection in the form of a cubic polynomial were used in the model.

In particular, there were considered the peculiarities of numerical modelling of coupled problems of gashydrodynamics flows and structural dynamics using the ANSYS Workbench, namely Fluent Flow and Transient Structural modules, which are combined with System Coupling. Also, the peculiarities of different approaches to multi-phase flow modelling are indicated. They are interesting not only by distribution of particles in the stream core, but also by the entrapped liquid film motion on the deposition surfaces.
\end{abstract}

Keywords: gas-dispersed flow, hydroaeroelasticity, preloading scheme, static load, stiffness matrix, wall film, boundary conditions, column vector, displacement.

\section{Introduction}

Considering the ratio of the specific energy consumption and separation efficiency of gas-disperse flows, the methods based on the use of inertia of particles (droplets) suspended in the gas-liquid flow [1], which is caused by a change in the motion direction of the gas-liquid flow, are optimal, and the deposit occurs on the surfaces of walls of the separation channels or deflection elements. The main drawback of this method is the secondary splashing, resulting from a possible increase of flow velocity to a critical value and disruption of the trapped liquid from the deposit surfaces, followed by the introduction of highly dispersed particles. Herein an increase also occurs in the hydraulic resistance, and therefore the actual task is to avoid these modes, which are realized in dynamic separation elements [2,3]. These elements work as an automatic control system, in which the regulating action is elastic forces, and the object of regulation is the hydraulic resistance. Under the fluviation directed into channels with elastic plates, hydrodynamic pressure is created, and in the walls of a channel the inertial stresses rise and change the surface form. In order to create the engineering method for calculation of separation elements of this type, it is necessary to solve a complex problem of the hydroaeroelasticity, which analytical solution is difficult, and sometimes even impossible without an introduction of simplifications and assumptions. Today, considering the high development of computer equipment, the software based on the finite volume method and the finite element method are used very often for its solving. But even they have their limitations. Let's consider the example of the ANSYS Workbench software suite, in which it is possible to solve the hydroaeroelasticity problem using the Fluent Flow (Fluent) and Transient Structural modules, which combination is carried out by means of System Coupling $[4,5]$. Taking into account the fact, that in the research of separation elements not only the plug flow particle distribution is of interest, but also the motion of the trapped liquid film along the deposition surfaces. 
Such calculation is possible in two cases, with a detailed solution of the wall area with the help of an exhausted volumetric grid, or by means of using the Eulerian wall film model $[6,7]$. Considering the peculiarity of the use of dynamic grids in these cases, the calculation will be computationally expensive, and therefore the optimization of channel design by constructing response surfaces and applying optimization algorithms is not appropriate in this case [6]. Therefore, it is important to develop a methodology for separation channels survey.

The solution of the optimal channel profiling problem usually presents considerable difficulties, therefore, in practice, approximate methods are used, based on physical concepts of the hydro- dynamically expedient distribution of velocities of the gas flow in the cross section and in the near-wall areas of the channel [8]. The separation channel is profiled by successive approximations, starting with the orienting constructions, according to the main requirements to the form and the ratio of the parameters of certain sections, the creation of conditions for inertial deposition of the disperse particles in the change of the flow direction of the solid phase flow, with the required average flow velocity of the passage sections, and then the lines embossing the channel shape are smoothed to prevent the combination of sections with a hopping in the radiuses of curvature in the flow. After preliminary construction of the curvilinear separation channel, we conduct the calculation of speed distribution in the channel computational region and on the walls, which limit it, and, if necessary, the channel form is further adjusted, in accordance with desired changes in the resulting velocity distribution. Particular attention during the profiling is required for the diffusive sections of the channels, where losses may be due to the separation of the flow. The possibility of separation limits the velocity gradient on the channel walls [9].

This paper considers the construction of dynamic separation elements, elastic elements of which are in the form of an inclined parabolic semi-cylinder [2] (Figure 1).

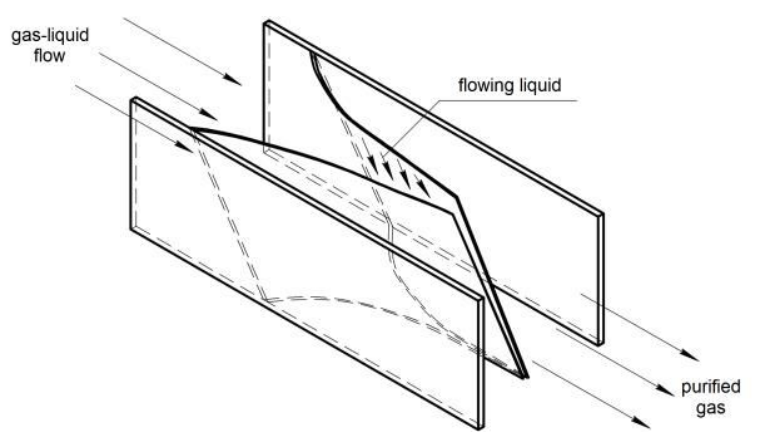

Figure 1 - Design scheme of the dynamic deflection element of the separation device

By virtue of the fact that fluviation changes the channels form that causes the change of flow parameters, static and dynamic hydroaerostrances will occur [10], among which the greatest interest will be represented by flutter and buffeting associated with the oscillating motion, when an essential role is played by elastic, aerody- namic forces and inertia forces. As a result of the vibration of the elastic deflection elements, there will also be oscillations of the gas-liquid flow, as a result of which the droplet may become concentrated in the liquid, and, consequently, the efficiency of the separation increases. Thus, due to the use of flutter or buffeting, the use of dynamic separation devices is possible. It should be noted that the acoustic coagulation of droplets is one of the traditional ways of improving separation efficiency. When applying acoustic oscillations with a certain frequency on the gas dispersion flow, intensive mechanical vibrations of highly dispersed suspended liquid droplets in the gas dispersion flow occur, resulting in a sharp increase in the number of their collisions. But the opposite effect of overlaying acoustic vibrations, in particular, the increase of the dispersion of the liquid phase, can be observed [11]. Therefore, it is necessary to know the specific frequency of oscillations in which there is a coagulation of suspended particles in the liquid. For the case of fluctuations of the gas-liquid flow caused by the action of sound waves, developed calculation methods that are well-matched with experimental results [12, 13].

Considering the foregoing, as in the case of acoustic vibratory coagulation, in order to achieve a positive effect on the oscillations imposed on the flow, it is necessary to know the optimum frequency thereof, which depends on the dispersion of the liquid phase. Whereas, the value of the flutter frequency depends on the geometric and elastic characteristics of the baffle elements. As can be seen from Figure 1, the elastic elements of the channel are predeformed, which means that they generate stresses, which increases their rigidity and consequently increases the flow rate at which the flutter can occur.

Therefore, the purpose of this paper is to conduct a static calculation of dynamic deflection elements of separation devices to research the previous stressed state.

\section{Results}

The previous deformed state of the dynamic deflection element of the separation device is modelled using analytical dependencies of the finite element method. In particular, the dynamic deflection element is represented by two-node finite element $e(i, j)$ with six degrees of freedom [14]. The design scheme of the preliminary deformed state of the dynamic deflection element of the separation device is shown in Fig. 2.

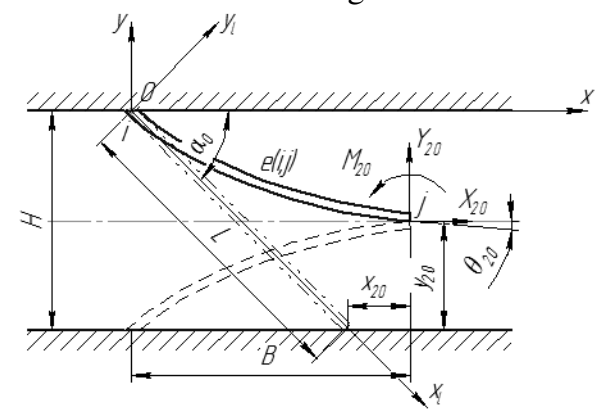

Figure 2 - Design scheme of the preloading dynamic deflection element of the separation device 
The local coordinate system $x_{l}-y_{l}$ is associated with the undeformed middle surface of the dynamic deflection element, the global coordinate system $x-y$ - with the fixed body.

The model provides the use of the deflection function of a median surface in the form of the cubic polynomial, presented in a matrix form:

$$
y_{l}\left(x_{l}\right)=\{\Phi\}\{U\}_{0 l},
$$

where $\{U\}_{0 l}=\left\{y_{10 l}, \theta_{10 l}, y_{20}, \theta_{20 l}\right\}^{T}-$ the column-vector of nodal displacement; $\{\Phi\}$ - row-vector of form orthogonal functions (interpolation matrix):

$$
\begin{aligned}
& \Phi_{1 y l}=1-3 \frac{x_{l}^{2}}{L^{2}}+2 \frac{x_{l}^{3}}{L^{3}} \\
& \Phi_{1 \theta l}=L\left(\frac{x_{l}}{L}-2 \frac{x_{l}^{2}}{L^{2}}+\frac{x_{l}^{3}}{L^{3}}\right) \\
& \Phi_{2 y l}=3 \frac{x_{l}^{2}}{L^{2}}-2 \frac{x_{l}^{3}}{L^{3}} \\
& \Phi_{2 \theta l}=L\left(-\frac{x_{l}^{2}}{L^{2}}+\frac{x_{l}^{3}}{L^{3}}\right)
\end{aligned}
$$

$L$ - - plate length; $y_{10 l}, y_{20 l}-$ nodes transverse displacement $i, j ; \theta_{10 l}, \theta_{20 l}$ - turn angles of cross-sections.

The index " 0 " indicates the consideration of the static equilibrium condition; the index " $l$ " corresponds to the local coordinate system; indices " 1 ", " 2 " indicate the belonging of a physical quantity to nodes $i, j$, respectively.

The connection between displacements of the deflection element and the forces that caused its deformation is determined by the matrix equation of the elastic equilibrium:

$$
[C]_{l}\{U\}_{0 l}=\{F\}_{0 l}
$$

where $\{F\}_{0 l}=\left\{Y_{10 l}, M_{10 l}, Y_{20 l}, M_{20 l}\right\}^{T}$ - vector of summarized forces, the elements of which are transverse forces $Y_{10 l}, Y_{20 l}$, applied in the nodes $i, j$, and the correspondent moments $M_{10 l}, M_{20 l} ;[C]_{l}-$ a local stiffness matrix whose elements are defined as second derivatives by the corresponding summarized displacements from the quadratic form of the plate deformation potential energy:

$$
C_{l i, j}=\frac{\partial^{2}}{\partial U_{l i} \partial U_{l j}} \int_{0}^{L} \frac{1}{2} E I\left[\frac{d^{2}}{d x_{l}^{2}}\left(\{\Phi\}\{U\}_{l}\right)\right]^{T} \frac{d^{2}}{d x_{l}^{2}}\left(\{\Phi\}\{U\}_{l}\right) d x,
$$

where $E$ - Young's modulus; $I=b^{3} h / 12--$ moment of cross section inertia for the rectangle cross section plate with height $b$ and thickness $h$.

The last formula does not consider the energy of the longitudinal deformation of the dynamic deflection element due to its sufficient flexibility, and the local stiffness matrix takes on the following form:

$$
[C]_{l}=\frac{E I}{l^{3}}\left[\begin{array}{cccc}
12 & 6 L & -12 & 6 L \\
6 l & 4 L^{2} & -6 L & 2 L^{2} \\
-12 & -6 L & 12 & -6 L \\
6 L & 2 L^{2} & -6 L & 4 L^{2}
\end{array}\right]
$$

In the global coordinate system $x-y$ matrix equation of elastic equilibrium has the form similar to the formula (3):

$$
[C]\{U\}_{0}=\{F\}_{0},
$$

where $\{U\}_{0}=\left\{x_{10}, y_{10}, \theta_{10}, x_{20}, y_{20}, \theta_{20}\right\}^{T}-$ the column vector of the nodal displacement $\{F\}_{0}=\left\{X_{10}, Y_{10}, M_{10}\right.$, $\left.X_{20}, Y_{20}, M_{20}\right\}^{T}$ - vector of summarized nodal forces with components along the axis $x\left(X_{10}, X_{20}\right), y\left(Y_{10}, Y_{20}\right)$ and moment $\left(M_{10}, M_{20}\right) ;[C]$ - the global stiffness matrix.

The transition from the local to the global matrix of rigidity is carried out due to the next formula:

$$
[C]=[T]^{T}[C]_{l}^{E}[T],
$$

where $[T]-$ transformation matrix:

$$
[T]=\left[\begin{array}{cccccc}
\cos \alpha_{0} & -\sin \alpha_{0} & 0 & 0 & 0 & 0 \\
\sin \alpha_{0} & \cos \alpha_{0} & 0 & 0 & 0 & 0 \\
0 & 0 & 1 & 0 & 0 & 0 \\
0 & 0 & 0 & \cos \alpha_{0} & -\sin \alpha_{0} & 0 \\
0 & 0 & 0 & \sin \alpha_{0} & \cos \alpha_{0} & 0 \\
0 & 0 & 0 & 0 & 0 & 1
\end{array}\right]
$$

$[C]^{E}{ }_{l}-$ the local stiffness matrix (5), expanded to size $6 \times 6$ by inputting values that correspond to longitudinal deformation:

$$
[C]_{l}^{E}=\left[\begin{array}{cccccc}
\frac{E A}{L} & 0 & 0 & -\frac{E A}{L} & 0 & 0 \\
0 & \frac{12 E I}{L^{3}} & \frac{6 E I}{L^{2}} & 0 & -\frac{12 E I}{L^{3}} & \frac{6 E I}{L^{2}} \\
0 & \frac{6 E I}{L^{2}} & \frac{4 E I}{L} & 0 & -\frac{6 E I}{L^{2}} & \frac{2 E I}{L} \\
-\frac{E A}{L} & 0 & 0 & \frac{E A}{L} & 0 & 0 \\
0 & -\frac{12 E I}{L^{3}} & -\frac{6 E I}{L^{2}} & 0 & \frac{12 E I}{L^{3}} & -\frac{6 E I}{L^{2}} \\
0 & \frac{6 E I}{L^{2}} & \frac{2 E I}{L} & 0 & -\frac{6 E I}{L^{2}} & \frac{4 E I}{L}
\end{array}\right]
$$

$A=b \cdot h$ - plate cross-section area

The case of a flexible deflection element allows not to consider in formula (9) the extension stiffness $E A / L$.

Taking account of the kinematic boundary conditions for the node $i\left(x_{10}=y_{20}=0 ; \theta_{10}=0\right)$ allows to expand the equation (6) of the elastic equilibrium of the dynamic deflection element into two matrix equations - separately for the nodes $i$ and $j$ :

$$
\begin{aligned}
& {[C]_{i}\{U\}_{0 j}=\{F\}_{0 i} ;} \\
& {[C]_{j}\{U\}_{0 j}=\{F\}_{0 j},}
\end{aligned}
$$


where the next descriptions are comprehended:

$$
\begin{aligned}
& {[C]_{i}=\frac{E I}{L^{3}}\left[\begin{array}{ccc}
-12 \sin ^{2} \alpha_{0} & -6 \sin 2 \alpha_{0} & 6 L \sin \alpha_{0} \\
-6 \sin 2 \alpha_{0} & -12 \cos ^{2} \alpha_{0} & 6 L \cos \alpha_{0} \\
-6 L \sin \alpha_{0} & -6 L \cos \alpha_{0} & 2 L^{2}
\end{array}\right] ;} \\
& {[C]_{j}=\frac{E I}{L^{3}}\left[\begin{array}{ccc}
12 \sin ^{2} \alpha_{0} & 6 \sin 2 \alpha_{0} & -6 L \sin \alpha_{0} \\
6 \sin 2 \alpha_{0} & 12 \cos ^{2} \alpha_{0} & -6 L \cos \alpha_{0} \\
-6 L \sin \alpha_{0} & -6 L \cos \alpha_{0} & 4 L^{2}
\end{array}\right] ;} \\
& \{F\}_{0 i}=\left\{\begin{array}{l}
X_{10} \\
Y_{10} \\
M_{10}
\end{array}\right\} ;\{F\}_{0 j}=\left\{\begin{array}{l}
X_{20} \\
Y_{20} \\
M_{20}
\end{array}\right\} ;\{U\}_{0 j}=\left\{\begin{array}{l}
x_{20} \\
y_{20} \\
\theta_{20}
\end{array}\right\} .
\end{aligned}
$$

The first equation of formula (10) allows to determine the internal forces and the moment that become in fixing the deflection element in the body of the separation device:

$\left\{\begin{array}{c}X_{10} \\ Y_{10} \\ M_{10}\end{array}\right\}=\frac{E I}{L^{3}}\left[\begin{array}{ccc}-12 \sin ^{2} \alpha_{0} & -6 \sin 2 \alpha_{0} & 6 L \sin \alpha_{0} \\ -6 \sin 2 \alpha_{0} & -12 \cos ^{2} \alpha_{0} & 6 L \cos \alpha_{0} \\ -6 L \sin \alpha_{0} & -6 L \cos \alpha_{0} & 2 L^{2}\end{array}\right]\left\{\begin{array}{c}x_{10} \\ y_{10} \\ \theta_{10}\end{array}\right\}$,

or in expanded form:

$$
\begin{aligned}
& X_{10}=-\frac{6 E I}{L^{3}}\left(2 x_{20} \sin ^{2} \alpha_{0}+y_{20} \sin 2 \alpha_{0}-\theta_{20} L \sin \alpha_{0}\right) ; \\
& Y_{10}=-\frac{6 E I}{L^{3}}\left(x_{20} \sin 2 \alpha_{0}+2 y_{20} \cos ^{2} \alpha_{0}-\theta_{20} L \cos \alpha_{0}\right) ; \\
& M_{10}=-\frac{2 E I}{L^{2}}\left(3 x_{20} \sin \alpha_{0}+3 y_{20} \cos \alpha_{0}-2 \theta_{20} L\right) .
\end{aligned}
$$

The second equation of formula (10) determines the internal forces that effect the free edge of deflection element as a result of its previous deformation (during the assemble).

Further on, the matrix $[C]_{j}$ is chosen as the main stiffness matrix of the system, and the second equation (10) the equation that determines the deformed state of the dynamic deflection element:

$$
\left\{\begin{array}{c}
X_{20} \\
Y_{20} \\
M_{20}
\end{array}\right\}=\frac{E I}{L^{3}}\left[\begin{array}{ccc}
12 \sin ^{2} \alpha_{0} & 6 \sin 2 \alpha_{0} & -6 L \sin \alpha_{0} \\
6 \sin 2 \alpha_{0} & 12 \cos ^{2} \alpha_{0} & -6 L \cos \alpha_{0} \\
-6 L \sin \alpha_{0} & -6 L \cos \alpha_{0} & 4 L^{2}
\end{array}\right]\left\{\begin{array}{l}
x_{20} \\
y_{20} \\
\theta_{20}
\end{array}\right\}
$$

or in expanded form:

$$
\begin{aligned}
& X_{20}=\frac{6 E I}{L^{3}}\left(2 x_{20} \sin ^{2} \alpha_{0}+y_{20} \sin 2 \alpha_{0}-\theta_{20} L \sin \alpha_{0}\right) \\
& Y_{20}=\frac{6 E I}{L^{3}}\left(x_{20} \sin 2 \alpha_{0}+2 y_{20} \cos ^{2} \alpha_{0}-\theta_{20} L \cos \alpha_{0}\right) \\
& M_{20}=-\frac{2 E I}{L^{2}}\left(3 x_{20} \sin \alpha_{0}+3 y_{20} \cos \alpha_{0}-4 \theta_{20} L\right)
\end{aligned}
$$

In the second equation (10) $\mathrm{i}$ and in the subsequent index " $j$ " is not specified, and the index " 20 " is replaced by " 0 ".
The final equation of an elastic equilibrium that determines the previous deformed state of a dynamic deflection element is the equation (6) for the following formulas of the global stiffness matrix [C] and the nodes displacement vectors $\{U\}_{0}$ and of the generalized forces $\{F\}_{0}$ in accordance with formula (14):

$$
\begin{aligned}
& {[C]=\frac{E I}{L^{3}}\left[\begin{array}{ccc}
12 \sin ^{2} \alpha_{0} & 6 \sin 2 \alpha_{0} & -6 L \sin \alpha_{0} \\
6 \sin 2 \alpha_{0} & 12 \cos ^{2} \alpha_{0} & -6 L \cos \alpha_{0} \\
-6 L \sin \alpha_{0} & -6 L \cos \alpha_{0} & 4 L^{2}
\end{array}\right] ;} \\
& \{U\}_{0}=\left\{\begin{array}{l}
x_{20} \\
y_{20} \\
\theta_{20}
\end{array}\right\} ;\{F\}_{0}=\left\{\begin{array}{c}
X_{20} \\
Y_{20} \\
M_{20}
\end{array}\right\} .
\end{aligned}
$$

The components of displacement $\{U\}_{0}$ are geometrically determined by the following correspondences:

$$
\begin{aligned}
& x_{20}=B-L \cos \alpha_{0} ; \\
& y_{20}=L \sin \alpha_{0}-\frac{H}{2} ; \\
& \theta_{20}=\operatorname{arctg}\left(\frac{H}{B}-\operatorname{tg} \alpha_{0}\right),
\end{aligned}
$$

where angle $\theta_{20}$ is determined approximatively for the parabolic plate bending; width $B$ for the deformed plate is in the range $\left(B_{\min }, B_{\max }\right)$ the limiting values of which are determined by the following correspondences:

$$
\begin{aligned}
& B_{\text {min }}=L-\frac{H}{2} ; \\
& B_{\text {max }}=\sqrt{L^{2}-\frac{H^{2}}{4}} .
\end{aligned}
$$

The least value $B_{\min }$ corresponds to the condition of the maximum possible deformation of the element, the largest value $B_{\max }$ is the rotation of the straight position element.

\section{Discussion}

The above-described static analysis mathematical model of the previously deformed dynamic deflection element of the separation device is reliable for the deflection forms of the middle surface that is interpolated by a quadratic or cubic polynomial. The transition to a two-dimensional formulation is substantiated for the case of setting external forces as a result of pressure field integrating along the plate height.

Further on, there is going to be proposed the ways of updating the initial parameters of this model and identification of unknown parameters - model's internal variables - in accordance with available experimental data and the results of numerical simulation based on the use of both the regression analysis and artificial intelligence systems, particularly artificial neural networks.

In general, the work's results may be aimed at improving the following tasks approaches: 
- the expansion of understanding of the theoretical foundations of separation processes of gas-dispersed systems in apparatuses with intensive hydrodynamic regimes;

- analysis of implementation of the physical conditions and formation models of gas-dispersed systems;

- analysis of mechanics and dynamics of the turbulent gas-dispersed flows;

- formulation of theoretical and practical problems of continuum mechanics and separation processes of the gas-dispersed flows;

- definition of the main methods to increase energy efficiency and the separation value of the modular separation devices.

\section{Conclusions}

The possibility of extending the dynamic separation elements application through the use of aero-hydroelastic effects, namely flutter, is revealed. For this purpose, a research of the static equilibrium condition of elastic deflection elements was conducted, because their pre-deformation has a sufficient effect on the flutter frequency. The stiffness of pre-deformed, their maximum and minimum possible deflections are determined.

Hereafter, it is planned to conduct research aimed at solving the stationary problem of aero-hydroelasticity of separation devices dynamic deflection elements. This problem can be solved in stationary and nonstationary formulations. Herewith, the analysis of the separation device deflection element deformation as the result of gas-liquid flow action should be based on the determining and further research of equation of plate flexural strain relative to the predetermined in this article static strained condition in accordance with the calculation scheme given in the Figure 2.

It is also necessary to analyze the dynamics of the separation device deflection element with respect to the position of the static equilibrium condition defined above, as well as plates dynamic equilibrium, including the determination of the optimal geometric dimensions that will provide the oscillation frequency of the element, which will coagulate the liquid droplets as a result of collision with the deflection elements of separation device.

\section{Acknowledgements}

Close cooperation between the Processes and Equipment of Chemical and Petroleum-Refineries Department and the Department of General Mechanics and Machine Dynamics allows carrying out the static calculation of the dynamic deflection elements for separation devices as a result of interdisciplinary research in chemical and petroleum industry within the following research commissioned by the Ministry of Education and Science of Ukraine: "Development and implementation of energy efficient modular separation devices for oil and gas purification equipment" (No. 0117U003931) [15].

This research project is aimed at im-proving the technology of inertia-filtering separation of two-phase flows, modelling of dynamic processes of separation of heterogeneous systems with the analysis of the vibration influence (acoustic oscillations) and combined heat and mass transfer, the development and implementation of energyefficient modular separation devices.

\section{References}

1. Liaposhchenko, O. O., Sklabinskyi, V. I., Zavialov, V. L., Pavlenko, I. V., Nastenko, O. V., \& Demianenko, M. M. (2017). Appliance of Inertial Gas-Dynamic Separation of Gas-Dispersion Flows in the Curvilinear Convergent-Divergent Channels for Compressor Equipment Reliability Improvement. IOP Conference Series: Materials Science and Engineering, Vol. 233. DOI: https://doi.org/10.1088/1757-899X/233/1/012025.

2. Liaposhchenko, O. O., Pavlenko, I. V., Nastenko, M. M., Usyk, R. Yu., \& Demianenko, M. M. (2015). Sposib vlovlyuvannya vysokodyspersnoyi kraplynnoyi ridyny z hazoridynnoho potoku [The method of capturing highly dispersed dropped liquid from the gas-liquid flow]. Certificate of the authorship, Ukraine, No. 102445 U, B01D 45/04 (2006.01). Sumy, Sumy State University, No. u201505124, bulletin No. 20 [in Ukrainian].

3. Liaposhchenko, O. O., Nastenko, M. M., Pavlenko, I. V., et al. (2016). Sposib vlovlyuvannya vysokodyspersnoyi kraplynnoyi ridyny $\mathrm{z}$ hazoridynnoho potoku [The method of capturing highly dispersed dropped liquid from the gas-liquid flow]. Certificate of the authorship, Ukraine, No. 111039 U, B01D 45/00 (2006.01). Sumy, Sumy State University, No. u201605061, bulletin No. 20 [in Ukrainian].

4. Sloboda, O., Korba, P., Hovanec, M., \& Pila, J. (2016). Numerical approach in aeroelasticity. Scientific Journal of Silesian University of Technology. Series Transport, Vol. 93, 115-122. DOI: https://doi.org/10.20858/sjsutst.2016.93.12.

5. Afanasyeva, I. N., \& Lantsova I. Yu. (2017). Numerical simulation of an elastic structure behavior under transient fluid flow excitation. Investigation of aerodynamic instability of a thin plate. MATEC Web of Conferences, Vol. 117, article No. 00099.

6. Zahed, P., Zhang, J.; Arabnejad, H., McLaury, B. S., \& Shirazi, S. A. (2017). CFD simulation of multiphase flows and erosion predictions under annular flow and low liquid loading conditions. WEAR, Issue 376, 1260-1270. DOI: https://doi.org/10.1016/j.wear.2017.01.111.

7. Yao, J., Yao, Y. F., Arini, A., Mciiwain, S., \& Gordon, T. (2016). Modelling air and water two-phase annular flow in a small horizontal pipe. Proceedings of the sixth international symposium on physics of fluids (ISPF6). International Journal of Modern Physics-Conference Series, Issue 42. DOI: https://doi.org/10.1142/S2010194516601587. 
8. Liaposhchenko, O., Nastenko, O., Pavlenko, I. (2017). The model of crossed movement and gas-liquid flow interaction with captured liquid film in the inertial-filtering separation channels. Separation and Purification Technology, Vol. 173, $240-243$. DOI: $10.1016 /$ j.seppur.2016.08.042

9. Nastenko, O., Liaposhchenko, O., et al. (2016). Mathematical modelling of separation process by coupled heat transfer in the inertial-filtering gas separator-condenser. Inżynieria i Aparatura Chemiczna, No. 2, 62-63.

10. Karintsev, I. B., \& Pavlenko, I. V. (2017). Hydroaeroelasticity: a textbook. Sumy, Sumy State University.

11. Brittle, S., Desai, P., Ng, W. C., Dunbar, A., Howell, R., Tesar, \& Zimmerman, W. B. (2015). Minimising microbubble size through oscillation frequency control. Chemical engineering research \& design, Issue 104, 357-366. DOI: https://doi.org/10.1016/j.cherd.2015.08.002.

12. Wu, Y. R., \& Wang, C. H. (2017). Theoretical analysis of interaction between a particle and an oscillating bubble driven by ultrasound waves in liquid. Chinese physics B, Volume 11, Issue 26, No. 114303. DOI: https://doi.org/10.1088/16741056/26/11/114303.

13. Go, D. B., Atashbar, M. Z., Ramshani, Z., \& Chang, H. C. (2017). Surface acoustic wave devices for chemical sensing and microfluidics: a review and perspective. Analytical methods, Volume 28, Issue 9, 4112-4134. DOI: https://doi.org/10.1039/c7ay00690j.

14. Pavlenko, I. V. (2006). Finite element method for the problems of strength of materials and linear theory of elasticity. Sumy : Sumy State University [in Russian].

15. Liaposhchenko, O. O., Demianenko, M. M., Lytvynenko, O. V., Ivanov V. O., Ostroga, R. O., Lytvynenko, A. V., Pavlenko, I. V., \& Dehriarov, I. M. (2017). Development and implementation of energy efficient modular separation devices for oil and gas purification equipment. Sumy, Sumy State University, No. 15.01.06-01.17/20.ZP, 2017-2020, State Reg. No. 0117 U003931. 